\title{
Clicker training increases exploratory behaviour and time spent at the front of the enclosure in shelter cats; Implications for welfare and adoption rates
}

\section{ABSTRACT}

The rescue shelter environment is known to be stressful for domestic cats, which can lead to them becoming less active, playful and exploratory as well as spending a long time hiding. Early adoption can prevent long term stress in shelter cats, but adopters often look at behaviour and friendliness as criteria when choosing a cat to rehome. This study aimed to test the efficacy of a clicker training intervention to promote behaviours indicative of improved welfare and increase the potential adoptability of cats in rescue shelters. Twelve cats were clicker trained over two weeks their behaviour and response to humans was recorded before and after the training schedule. Cats showed significantly more exploratory behaviour, a decrease in inactivity and spent more time at the front of their enclosures after training. Four of the cats which failed the human approach test initially, passed it after training but this result was nonsignificant. Clicker training may be a simple and rapid way to improve welfare and adoptability in rescue cats.

\section{Introduction}

The welfare of domestic cats may be at risk due to owners relinquishing their animals to rehoming centres and shelters (Casey et al., 2009). Reasons for relinquishment include health problems such as allergies to cat fur, changes in circumstances (such as a move to rented accommodation not allowing pets), and behavioural problems (Scarlett et al., 1999; Shore et al., 2003; Casey et al., 2009). Stray and feral cats also account for a large proportion of the residential shelter population worldwide (Salman et al., 1998; Levy and Crawford, 2004; Alberthsen et al., 2013). The shelter environment is often stressful for cats, due to a combination of novel stimuli, healthcare and handling procedures which cats perceive as aversive, and close proximity to other felines (as cats tend to be solitary, territorial and intolerant of unrelated conspecifics (Ley, 2015)). Additionally, there are usually restrictions on space and lack of control over the environment (Dybdall et al., 2007; Gourkow et al., 2014a,b).

This stress can manifest in a variety of ways such as aggression to humans or conspecifics, repetitive behaviours such as pacing or over grooming and stress-related health problems, such as upper respiratory tract infections exacerbated by the immunosuppressive effects of high cortisol levels (Tanaka et al., 2012). Cats generally spend a proportion of their activity budget inactive, but inactivity is seen to increase with long shelter stays, along with agonistic behaviour (Gouveia et al., 2011). Inactivity may be related to stress, as cats more frequently respond to aversive environments by inactivity rather than behavioural abnormality (Rochlitz, 2000), although it is difficult to quantify how much inactivity is indicative of reduced welfare states. Inactivity in enclosed environments also predisposes cats to health problems such as obesity (Scarlett et al., 1994; Selman, 2017) and diabetes mellitus (Slingerland et al., 2009). Furthermore, activity levels directly affect adoptability, with active cats having greater adoption rates (Fantuzzi et al., 2010).

Therefore, decreasing inactivity in shelter cats would be beneficial for a number of reasons. Long term residents of shelters may experience ongoing stress as well as being at increased risk for euthanasia due to overcrowding (Alberthsen et al., 2013; Salman et al., 2000; Bartlett et al., 2005), therefore early adoption is the goal of many rescue shelters. There are many factors which may influence the adoptability of an individual cat, such as age, breed, sex, pelage and health status (Lepper et al., 2002). Behaviour and temperament may also play a large role in the likelihood of a cat being rehomed, with many potential adopters citing cats' willingness to interact with them, friendliness and a happy disposition as a major influencing factor in their decision-making process (Dybdall and Strasser, 2014; Gourkow, 2001; Gourkow and Fraser, 2006). Unfortunately, previous research suggests that the length of time cats spend within shelters may contribute to a reduction in activity levels, with long-stay residents demonstrating greater levels of inactivity and time spent engaging in negative behaviours compared to cats who had spent less time in that environment, perhaps due to their stress levels rising over the duration of their stay (Gouveia et al., 2011).

Therefore, adoptability is directly linked to welfare, as early adoptions can prevent the cumulative effect of the stressful shelter environment. Such research highlights the fact that efforts should be made to reduce the amount of stress experienced by shelter cats, whilst simultaneously reducing the length of time cats spend within a shelter before being rehomed (Gouveia et al., 2011). According to Gourkow and Fraser (2006), consistent and positive handling experiences with humans may directly correlate with lower stress scores and a reduction of fear-induced aggression in shelter cats. This is supported by Gourkow et al. 
(2014a,b) who discovered that regular gentle, positive handling experiences increased behavioural indicators of contentment and reduced physiological indicators of stress in shelter cats significantly.

In recent years operant conditioning using positive reinforcement based training methods such as clicker training have become recognised as a valuable method of managing captive species (Melfi, 2013) and can facilitate animals' voluntary cooperation with otherwise aversive handling procedures (Ward and Melfi, 2013), leading to a decrease in stress (Savastano et al., 2003). Clicker training is also a form of enrichment as it encourages animals to be more physically active as well as stimulating them mentally whilst promoting positive interactions with humans (Laule and Desmond, 1998). However, Westlund (2014) stresses that training may only be considered a form of enrichment if meets certain criteria, such as empowering the animal to feel in control over its environment and facilitating the animal to perform its natural behaviours. Ward and Melfi (2013) add that such positive interactions may serve to reduce animals' feelings of fearfulness towards humans which reduces their stress levels and therefore improves welfare. Captive felines are particularly susceptible to high stress levels (Shepherdson et al., 1993) and therefore may benefit from training programs that encourage species-appropriate behavioural repertoires in an environment where they have limited sensory stimuli (Wells, 2009).

Although domestic dogs undergo regular training and behavioural modification within shelters to alter their behaviour to be more desirable to adopters (Luescher and Medlock, 2009) this practise is rarely, if at all, implemented amongst shelter cats. Cats can be clicker trained to perform novel behaviour (Kogan et al., 2017), but it is not clear whether training can reduce behavioural signs of stress and increase behaviours likely to result in early adoption. Shelter staff have limited time available for training so a method which is easy to use and takes little time, while demonstrating maximum effectiveness would be the most likely to make a practical difference to the welfare and adoptability of rescue cats.

In this study, we tested the hypothesis that 10 min sessions of clicker training 3 times per week for 2 weeks would: i Increase exploratory behaviour in shelter cats ii Increase playful behaviour iii Reduce inactivity iv Increase the amount of time spent at the front of the cage $v$ Improve cats' initial responses to unfamiliar humans, measured by the human approach test (Arhant and Troxler, 2017) 2. Methodology The project was approved by Oxford Brookes University and RSPCA Oxfordshire. Due to the test subjects residing in a rehoming centre and awaiting adoption, it was important that any research being carried out would not disrupt the rehoming procedure or compromise their chances of finding new homes. Therefore, normal adoption proceedings continued throughout the course of the project and a number of subjects were rehomed before the project had run its full course. As one of the primary goals of the study was to increase the cats' adoptability, it would undoubtedly be unethical to halt adoption proceedings for the sake of the research, regardless of whether it was temporarily or otherwise.

All of the subjects were monitored carefully throughout to ensure they were not put under any undue stress or that their welfare was compromised at any time. The training operated under the principles of positive reinforcement as they were rewarded with food for correct behaviour (Fisher, 2009). The project itself was designed to be as minimally stressful as possible. Research was carried out in such a way that the subjects were not confined to a small area in which they would be unable to remove themselves at any point should they wish to. The experimenter remained an appropriate distance away from the individual subjects at all times and remained vigilant of any behavioural indicators of stress, such as vocalisation or attempting to hide (Kessler and Turner, 1997). Particularly fearful cats were not handled by the experimenter, making their involvement with the project completely optional, allowing them control over their environment and a giving them a choice about whether to approach the investigator or not.

Prior to any research taking place, it was decided that if at any point any of the subjects displayed behaviour indicative of high stress as a direct result of the project, they would be permanently removed from all further research. Research was conducted within the RSPCA's Oxfordshire rehoming centre, located at Common Leys farm in Hailey, Oxfordshire, UK. The cats are held in a cattery which is located within a multi-use barn situated on the college campus. The cattery consists of 9 individual holding pens with a walk-way running from one end to the other with two access points at opposite ends (Fig. 1). 


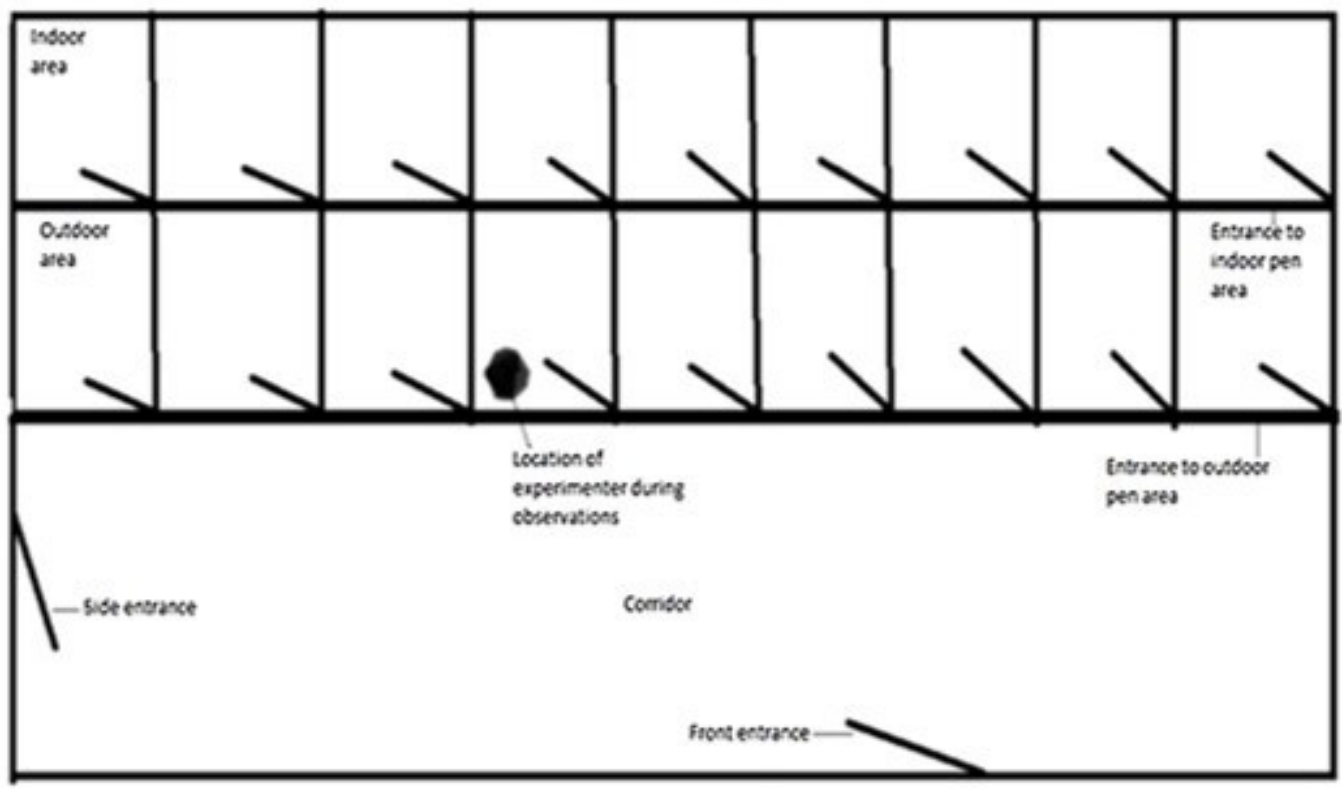

Figure one - layout of the catterey and position of the observer

The holding pens feature an indoor area consisting of four opaque sides with access via a cat flap to an outdoor area with opaque sides and a door made of woven wire mesh which opens onto the walk-way area. The cattery itself is primarily made up of PVC material, for means of easy cleaning and sterilisation. The cats are obscured from one another's view whilst within their pens but they are audible to one another. The indoor area of the pens consists of a floor and a raised shelf from which the cats have visual access to the outdoor area via a window and contains a heater and bedding. Litter trays and scratching posts are usually placed in the outdoor area. Each cat is provided with a selection of toys which are variable in nature, due to the fact that some come from cats' previous residences and others are donated by the public.

The cattery is maintained by staff and students who enter to perform routine husbandry duties twice daily, as well as by RSPCA volunteers who attend to the cats multiple evenings a week for socialisation, a practise which continued throughout the experiment, due to concerns that temporarily suspending socialisation might negatively impact the cats' welfare. The barn in which the cattery is situated also contains the college students' personal lockers and so individuals pass through multiple times per day to deposit and collect their belongings.

The cattery is designed to house 9 cats at a time, however, during busy intake periods as many as 4 cats were observed to be contained within a single pen at a time. For the purpose of standardising this experiment, only cats housed singly were included. Due to the relatively small size of the cattery, the number of cats available for training was limited. Therefore, all of the cats residing in the cattery at the time of the study were subjected to clicker training, with no discrimination based on factors such as breed, age, sex or temperament. However, a number of cats were ultimately excluded from the study, due to some being rehomed during the process and one becoming a veterinary in-patient due to an ongoing illness. The final sample used in the study consisted of 12 cats (eight males and four females) of a variety of ages, of which ten were relinquished by owners and two were former strays (Table 1). Fig. 1 shows the layout of the cattery and the position of the observer.

\section{Table 1}

The sex, age, provenance, and duration of stay of the 12 cats included in the study 


\begin{tabular}{|c|c|c|c|c|c|}
\hline Cat ID & Sex & Age (years) & $\begin{array}{l}\text { Owner } \\
\text { relinquished } \\
(\text { OR)/Former } \\
\text { stray (FS) }\end{array}$ & $\begin{array}{l}\text { Duration of stay } \\
\text { (prior to initial } \\
\text { behavioural } \\
\text { observation) }\end{array}$ & $\begin{array}{l}\text { Cats whose } \\
\text { score } \\
\text { improved in } \\
\text { the HAT }\end{array}$ \\
\hline 1 & M & 1 & OR & 3 days & \\
\hline 2 & M & 4 & OR & 2 days & \\
\hline 3 & M & 1.2 (approx) & OR & 8 days & \\
\hline 4 & M & 3 & OR & 7 days & $y$ \\
\hline 5 & M & 9 & OR & 19 days & y \\
\hline 6 & M & 11 & OR & 21 days & \\
\hline 7 & M & 2 (approx) & FS & 7 days & $y$ \\
\hline 8 & M & 2-4 (approx) & FS & 9 days & \\
\hline 9 & F & 5 & OR & 3 days & \\
\hline 10 & F & 6 & OR & 2 days & \\
\hline 11 & F & 10 & OR & 25 days & $y$ \\
\hline 12 & F & 4 & OR & 2 days & \\
\hline
\end{tabular}

\subsection{Observing and recording behaviour}

Behaviour was observed by the researcher and also using a Samsung Galaxy S8 mobile phone camera that was positioned on the floor of the pen with the entire pen within frame by the handler and held in place with the use of inanimate objects that were at their disposal within the cattery. Four behavioural categories were chosen based on studies demonstrating reduced activity levels and a lack of playful and exploratory behaviour as stress indicators (Carlstead et al., 1993a,b; Uetake et al., 2013). The categories were exploratory behaviour, inactivity, play and other. The "other" category was reserved for generic behaviours that did not fall under the former three and are neither indicative of stress or contentment in cats, such as non-excessive grooming, clawing or stretching, eating, drinking and defecation (Stanton et al., 2015). Each cat's behaviour was observed and recorded for a total of $10 \mathrm{~min}$ before and after training, using an automated timer to mark the end of each session. The handler was present within the pens during filming, however they remained as still as possible and did not engage with the cats in any way. The total duration of each of the behavioural categories (exploring, playing, inactive and other) was recorded. Once each cat had been individually recorded for a total of ten minutes, the footage was analysed in order to calculate the precise duration of which any of the aforementioned behaviours occurred. This double analysis was intended to improve accuracy in recording the behaviour.

Behaviours were identified as follows: Exploratory behaviour - the cat moves purposefully around its environment in an attentive manner whilst investigating surfaces or objects by sniffing them or manipulating them with their head, body or paws (Stanton et al., 2015). Play behaviour - the cat interacts with objects in a way in which harm is unintended in a playful context with an absence of any behaviours indicative of aggression, such as hissing or growling (Stanton et al., 2015). Inactivity - cats are stationary in either a sitting, lying or standing position, either sleeping or awake (Stanton et al., 2015). Additionally, during the ten-minute observation period the amount of time each cat spent at the back of the cage (BOC) and the front of the cage (FOC) was quantified before and after training. For the purpose of the experiment, their position in the cage was defined by whichever side of the door separating the two areas of the cage they were situated in that instance. For instance, for a cat to be considered to be at the front of the cage, the entirety of its body must be positioned in the outdoor area of their enclosure.

The Human-Approach Test (HAT) was developed by Arhant and Troxler (2017) as a means of measuring cats' reaction to humans in the stressful environment of a shelter. The test is performed by a handler approaching the individual cats slowly and with smooth, fluid motions, avoiding direct eye contact at all times and then presenting their hand to the cat, holding it approximately $20 \mathrm{~cm}$ away from their face. The cat is classed as "contact possible" (CP) if they investigated the hand, such as by sniffing or head rubbing, or if they simply did not withdraw away from it. The cat is classed as "no contact possible" (NCP) if they appear to freeze at the presentation of the hand, withdraw away from it or display any signs of aggression.

The cats in this study were presented with the human approach test before and after training and the number of cats which were contact possible was recorded 2.2. Clicker training Clicker training commenced 
the day after the behavioural observation had taken place and the cats' position in their cages and their HAT score had been recorded. The first two training sessions consisted of charging the clicker to become a conditioned reinforcer by clicking in close proximity to the individual subject before immediately presenting a food reward (Kelleher and Gollub, 1962). This was then repeated several times throughout the session, until the cat began to anticipate the arrival of the food upon hearing the click (Pryor, 2009).

The aforementioned food reward consisted for the most part of commercially sold Dreamies or Whiskas cat treats. However, research suggests that acquisition speed in training positively correlates to the utilisation of a variety of food types (Polín and Pérez, 2017). Therefore, the trainer would at times deliver food of higher value to the cats, such as fresh chicken or ham on a variable-interval schedule of reinforcement. The food rewards were only delivered in very small portion sizes at a time, to ensure the cats were not being overfed as well as to limit the chance of them becoming satiated and their motivation levels decreasing before the end of the training sessions (Finan and Taylor, 1940). Following on from those initial sessions, the distance was gradually increased between the trainer and the cat, so that the cat had to travel towards the trainer in order to receive the reward. The trainer added a vocal cue by calling the individual cats' name and then clicked to mark the desired behaviour when the cat approached them to within a close enough proximity that they could outstretch their arm to deliver the food with ease. In the case of extremely fearful cats that would obscure themselves from the trainers' sight within dome beds/boxes, hiss or lash out at the trainer when approached, food was gently tossed towards the cat so that it landed within close proximity of the front of their bodies, but at a distance which would require the cat to travel forwards in order to reach it.

The trainer observed the cat for as long as required, periodically calling the cats' name, then when the cat would make any sort of movement towards the food the trainer clicked to capture the behaviour. The trainer then shaped this behaviour by increasing the duration between the movement of the cat and the click until the cat felt finally confident enough to approach the outdoor area of the pen, from then on the training sessions could continue as with the more confident subjects. The aim of the latter sessions was to use shaping to encourage the cats into the outdoor area of the pen and as close to the front as possible.

The final aim of the training sessions was for the subjects to travel from wherever they were positioned at the start of the session to the front of the outdoor area when their name was called by the trainer. The training schedule ran through its entirety throughout the course of two weeks. Two days after the final session, the cats' behaviours and position in cage were re-observed and recorded and they were once again subjected to the Human-Approach Test to record any changes that may have occurred. The HumanApproach Test was repeated using a handler whom which the cats had not previously encountered so that they were completely unfamiliar with them, to eliminate the possibility that the cats' HAT score may have improved when re-tested due to the fact that they had become accustomed or habituated to the individual handler, rather than becoming less fearful of humans approaching in general (Arhant and Troxler, 2017).

Paired t-tests were carried out on the total duration of each behaviour and the amount of time at the front of the cage, before and after training. We used McNemar's test in a $2 \times 2$ contingency table on the number of cats that were contact possible before and after the training. All data were analysed in Minitab 18.

\section{Results}

5

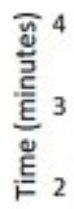

1
Mean time spent in exploratory behaviour

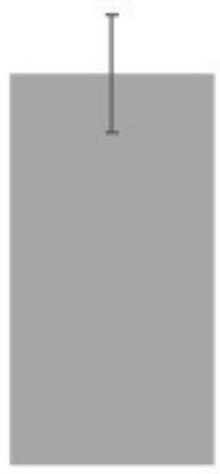


9

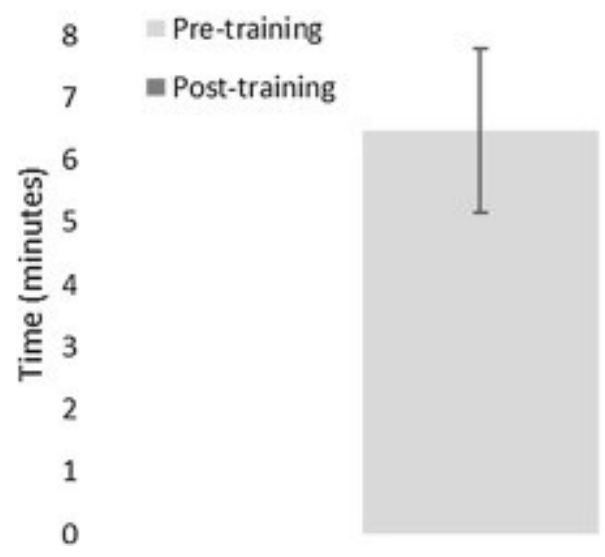

Time spent at the front of the cage

10

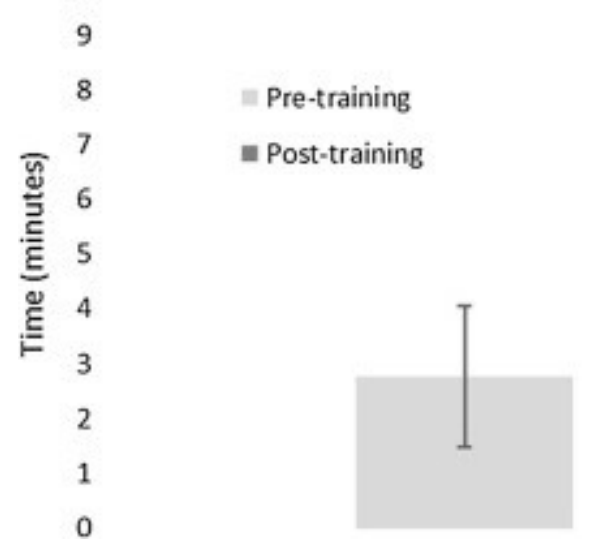

Number of cats scrored "contact possible"

12

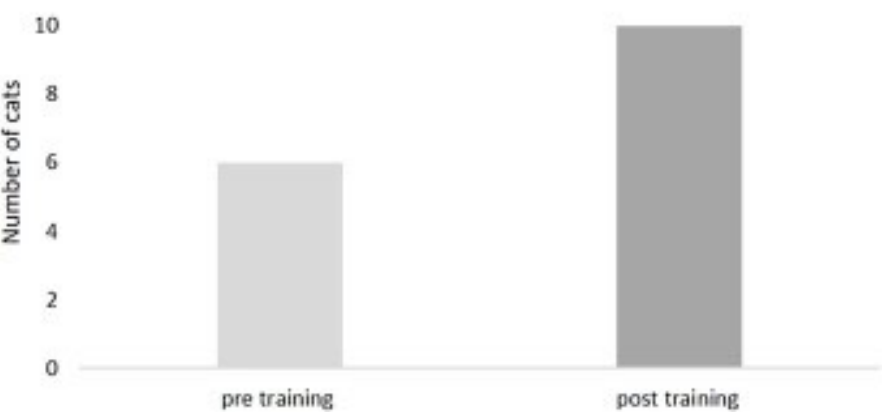

Cats spent significantly more time in exploratory behaviour after training (Fig. 2$),(n=12 ; T=4.33 ; p=$ 0.001 ). Although the mean time spent playing increased slightly after training, due to the high variability of this behaviour between subjects, no significant difference in play was found $(n-12 ; T=-1.08 ; p=0.302)$. There was a significant decrease in mean time spent inactive (Fig. 3) $(n=12 ; T=4.09 ; p=0.002)$. Cats spent significantly more time at the front of the cage after training (Fig. 4$)(n=12 ; T=-4.67 ; p=0.001)$. The results of the human approach test showed that more cats were scored as "contact possible" rather than "no contact possible" after training (Fig. 5). Before training six out of 12 cats were scored contact indicated no significant difference $(p=0.125)$. 


\section{Discussion}

\subsection{Exploratory behaviour}

Prior to the clicker training sessions, the majority of the subjects demonstrated a considerable lack of exploratory behaviour, with several of the subjects spending absolutely no time exploring during the tenminute observational sessions whatsoever. The results of this study showed that there was a significant increase in exploratory behaviour post-clicker training, which supports our prediction that clicker training will increase exploratory behaviour. These results suggest that the clicker training had a positive effect on the cats' welfare, at least to some degree, as previous research states that a reduction in levels of exploration is indicative of heightened stress levels in felines (Carlstead et al., 1993a,b).

Additionally, the fact that clicker training appeared to increase the cats' locomotive behaviour and their level of engagement with their surroundings fulfils Westlund (2014)'s criteria by which animal training can be considered enriching, as it would appear to have given the cats more behavioural choices and facilitated their engagement in species appropriate repertoires, as cats are by nature highly exploratory animals (Kemp and Kaada, 1975). In a rehoming environment, cats are restricted spatially, therefore it is important to provide environmental enrichment to encourage optimum spatial utilisation (Ross et al., 2009).

The results of this study suggest that clicker training may be an effective way of achieving this.

\subsubsection{Playing}

Play behaviour was low before training and did not significantly increase after training. Some consider play to be a particularly important behavioural repertoire in captive felines, as it facilitates the rehearsal of predatory hunting behaviour that these animals would naturally perform in the wild (Biben, 1979). Additionally, previous research suggests that potential adopters are more drawn to cats in rehoming centres that appear playful (Gourkow, 2001). In this study the experimenter simply sat stationary within the cats' holding pens, remaining quiet and still, without directly engaging with the cats in order that they did not deliberately facilitate or prompt any instances of behaviours that were being recorded. The outside areas of the pens in which the experimenter was stationed were relatively unenriched, many of them containing only a litter tray and a food and water station. Toys were mainly contained within the indoor area of their pens, providing the cats with little opportunity to play in the outdoor area of their pens (McCune et al., 1995). Therefore, it is reasonable that instances of playfulness were low during this study.

Should the experimenter have directly engaged with the cats in a playful manner during the behavioural observations, such as by dangling a toy in front of them for example, it could be hypothesised that this would have incited a much higher frequency of playfulness, however this was intentionally avoided in order that the results be unbiased.

\subsubsection{Inactivity}

In the initial behavioural observation prior to clicker training many of the cats spent a considerable amount of time inactive, but this behaviour showed a substantial decrease after training. As inactivity may be a reaction to stress, and is certainly a predictor of poor health states and lower adoptability, the reduction in inactivity seen may be indicative of improved welfare in the cats.

\subsubsection{Other behaviours}

Behaviours which fall under the category of 'other' for the purpose of this study include all behaviours which cannot be placed under the categories of exploration, playfulness or inactivity. These include maintenance behaviours which, providing they are not performed to excessive levels, are neutral in nature, such as eating, drinking, grooming and defecating (Stanton et al., 2015).

The results of this study indicate that there was a significant increase in other behaviours post-training $(p=$ 0.015). Unfortunately, due to logistical constraints the authors of this study were unable to further investigate the significant increase in "other behaviours" to determine which particular behaviours increased and for what reason they may have done so. The experimenter noted that they witnessed an increase in the amount of time a number of the cats spent eating during the ten minute behavioural observations, however these instances were not quantified and therefore there is no statistical evidence to testify to this. 
However, this could provide an interesting opportunity for further research as cats are known to be susceptible to stress-related anorexia when confined to rehoming centres (Amat et al., 2016) and therefore, should it be hypothesised that clicker training can increase cats' feed intake, it could perhaps be recommended as a routine aspect of husbandry to be utilised amongst shelter staff.

Previous research has focused on the effect of housing, husbandry practises and positive human interactions on body condition of shelter cats (Gourkow et al., 2014a,b; Gourkow and Fraser, 2006; Tanaka et al., 2012) however, to the authors' knowledge, no studies have focused on the effects of training on feed intake or body condition in domestic cats.

\subsubsection{Position in cage}

For this study, one of the primary objectives was to encourage the subjects to come to the front area of their pens/cages in response to the presence of a human. The reason for this was that the cattery in which the study took place is designed as such that when a human enters through the main door they are situated within a walkway that runs from one end of the cattery to the other and from this area they are able to view the outdoor areas of the individual pens in which the cats are held. The indoor areas of the pens are separated by doors which are kept fastened shut by hinges wherein the cats are hidden from the view of the humans. Many potential adopters claim that the degree to which a shelter cat is willing to interact with them would highly influence their decisions (Gourkow, 2001) and, previous to the commencement of the study, it was observed that many of the cattery's residents would spend the majority of their time obscured from view in the back of their cages. Therefore, the assumption was made that these individuals may be being overlooked by adopters, in comparison to those that were seen to travel directly to the front of their cages in response to the arrival of visitors to the cattery.

Based on these assumptions, the training protocol for the study was designed to lure the cats to the outdoor areas of their cages, with the end goal being that they would travel to the front of their cages in response to the arrival of a human. The results of this study show that there was a significant rise in the amount of time the cats spent at the front of the cage post-training in comparison to pre-training which supports the study's original hypothesis. The experimenter observed that, pre-training, a number of the cats spent the entirety of the ten minute observational session in the indoor area of their pens hiding and either partially or entirely obscured from view. For cats, hiding behaviour is often a reaction to high stress levels, therefore cats should always be provided with the opportunity to hide (Vinke et al., 2014), however one of the aims of this study was to reduce the cats' stress levels in order for them to better cope in their environment, so that they may willingly and voluntarily travel to the outdoor area of their pen in the presence of humans.

For example, one of the subjects (N7) was positioned at the back of their cage for the entire duration of the behavioural observation pre-training, and was also inactive for the majority of the session. In contrast, however, the subject was observed to be positioned at the front of the cage for 9.86 min during the posttraining observation, suggesting a significant decrease in the animal's stress levels (Vinke et al., 2014). These results suggest that clicker training may also serve as a method of encouraging optimum spatial utilizations of enclosures by captive animals whilst increasing their activity budget (Mallapur et al., 2002). R.A. Grant, J.R. Warrior Applied Animal Behaviour Science 211 (2019) 77-83 81 4.1.5. Human-approach test The Human-Approach Test was designed by Arhant and Troxler (2017) to assess cats' responses to humans in a measurable, repeatable and scientifically valid manner. In their study, they found that positive HAT scores in cats correlated with positive interactions with shelter staff, which inspired this study's hypothesis that clicker training would improve cats' HAT score, as training sessions which utilise positive reinforcement techniques are considered to be a pleasurable experience for animals (Miller, 2001; Herron et al., 2009; Pryor, 2009).

The results of this study show that there was an improvement in the subjects' HAT scores following the training sessions. In fact, at the end of the study only 2 subjects (N6, N10) were classified as "no contact possible" by the experimenter, which represents a momentous advancement compared to the results pretraining. It should be noted, however, that a cat is not only classified as "no contact possible" based on whether it displays aggressive behaviour towards a handler, such as scratching or biting, this classification may simply be applied if the cat in question withdraws or freezes when presented with the experimenters hand (Arhant and Troxler, 2017). Therefore, N6 and N10 may not necessarily have been experiencing high stress levels during the test in response to the handler, however it is recommended that efforts be made in cases such as this to improve individuals response to humans in order to eliminate any instances of fear or 
aversion as this can have an effect on animals wellbeing (Rushen et al., 1999). These results indicate that clicker training may improve cats' adoptability, as adopters tend to prefer cats that appear friendly and willing to interact with them (Gourkow, 2001), therefore by improving cats' response to the unfamiliar handler within the context of the study, it may be assumed that their response to potential adopters visiting the cattery will also be positive. McNemar's test on the number of cats "contact possible" before and after training failed to give a significant outcome. This is probably due to the small sample size and half the cats already being "contact possible" before training and only four cats moving from "no contact possible" to "contact possible".

However it is clear that several cats moved in a positive direction, and no cats in the reverse direction. Further research using a larger sample could expand on this study on the effect of clicker training on the human approach test. 4.2. Limitations 4.2.1. The cattery One of the main limitations of the study was that the cattery was still actively operating as a rehoming and adoption centre during the time that data collection was taking place, there was a lack of environmental control. For instance, on several occasions during training the sessions would be momentarily interrupted by arriving staff or volunteers, at which time the training would be paused and picked up at a later instance to ensure there were minimal opportunities for the cats to become distracted. This was also to ensure that the novel behaviours being reinforced in the training sessions were brought under stimulus control, and the subjects would only be rewarded for performing a behaviour when prompted by the experimenters' cue, rather than any other sort of signal offered by any other visitors to the cattery (Lindsay, 2013).

It was also observed that as the study took place during the winter months, during times of extreme weather conditions the subjects would momentarily appear to be visibly distracted by noises emitting from outside the cattery, such as heavy rain or strong winds. However, due to the open design of the barn there was little that could be done by the experimenter to counteract these effects. A small sample size $(N=12)$ was a limiting factor in this study due to a combination of factors such as a quiet period in terms of admissions to the cattery and dropouts throughout the time of data collection due to subjects being rehomed or admitted to veterinary surgeries, only 12 cats remained at the end of the training schedule. Due to the relatively high rate of adoption that was observed prior to data collection, the training schedule was designed to take place over a relatively short length of time, with training sessions taking place 3 times over the course of 2 weeks.

However, previous research into the effects of the duration and frequency of obedience training on levels of acquisition in dogs suggests that dogs trained once a week learn more effectively than those trained more frequently (Meyer and Ladewig, 2008; Demant et al., 2011). Therefore it could be speculated that the efficacy of the study could have been improved had the training been less frequent, however the focus of the study was to determine whether clicker training had a positive effect on the subjects' welfare, as opposed to the rate in which they were able to learn novel behaviours. However the study has showed that only a small amount of time needs to be spent on clicker training the cats to produce a large increase in desirable behaviours indicative of good welfare and likely to secure early adoption.

Additionally, another factor that must be considered is the possibility that the cats' became less stressed over the course of the study because the novelty of their environment had decreased throughout the duration of their stay. Many of the cats had only recently been admitted to the cattery prior to the preliminary behavioural observation and human-approach test, suggesting that poor performance in any of the aforementioned categories may have been due to high stress levels resulting from their arrival at the cattery. Previous research regarding boarding catteries suggest that cats experience high levels of stress postarrival, which rapidly decline over the course of 14 days of confinement (Kessler and Turner, 1997). Similar results are seen in dogs admitted to rehoming centres, with high levels of cortisol ratios being recorded on the day of their admittance which gradually decline over the course of 14 days (Hiby et al., 2006). However, looking at the individual results of the human approach test, where the four cats which improved from no contact possible to contact possible were also the cats with the longest duration of stay and some of the oldest cats in the study.

Therefore we consider that the results shown, at least in the HAT test, were not simply due to habituation to the shelter environment. Gourkow et al. $(2014 a, b)$ also discovered that regular positive handling experiences with humans had a positive influence on the health of shelter cats and reduced instances of fearful or aggressive behaviour. Therefore, it could be suggested that any observed behavioural changes in the subjects of this study manifested because the experimenter regularly engaged in positive interactions with the subjects during the training sessions, by handling them gently and positively reinforcing their 
behaviour, rather than as a result of the clicker training specifically. Perhaps future research into the effect of clicker training on the welfare of shelter cats may benefit from utilising two groups of test subjects; one of which is clicker trained and the other simply experiencing gentle handling in order to compare the any behavioural changes between the two. As the clicker training and the behavioural observation were performed by the same person it is possible that the cats' behaviour changed as a result of a classically conditioned association of the handler with a food reward. This cannot be ruled out, and we suggest that in a future study the behavioural observations are done by video or an unfamiliar individual. Nevertheless, the HAT was carried out by an unfamiliar individual and four cats out of the six who were not contact possible had changed their behaviour, suggesting that clicker training, not conditioned association with food, was the causative agent. Despite some limitations of the methodology due to constraints outside our control, the study provides a useful starting point for further research into clicker training as an intervention in shelter cats. The research can be used as a pilot study, suggesting that clicker training interventions may be a suitable, cost effective way of improving welfare and adoptability.

Further research will be carried out to explore the effects of the intervention in more detail and in various contexts. 5. Conclusion The aim of this study was to investigate whether clicker training has a positive effect on the welfare of cats confined to a rehoming centre, R.A. Grant, J.R. Warrior Applied Animal Behaviour Science 211 (2019) 77-83 82 measured by behavioural scores and the human approach test. Improvements were seen in the amount of time the subjects spent exploring, engaged in other behaviours, positioned at the front of their cages and individuals' HAT scores, whilst inactivity decreased. Due to a number of limitations to the study, the author suggests that it may prove beneficial to repeat the study using a more refined methodology, utilising a larger number of test subjects in a more controlled setting. Based on the results of this study, further research into the welfare of cats that have been clicker trained compared with those who have only experienced traditional handling by staff and volunteers within a shelter may provide enlightening results.

\section{References}

Alberthsen, C., Rand, J.S., Bennett, P.C., Paterson, M., Lawrie, M., Morton, J.M., 2013. Cat admissions to RSPCA shelters in Queensland, Australia: description of cats and risk factors for euthanasia after entry. Aust. Vet. J. 91 (1-2), 35-42.

Amat, M., Camps, T., Manteca, X., 2016. Stress in owned cats: behavioural changes and welfare implications. J. Feline Med. Surg. 18 (8), 577-586.

Arhant, C., Troxler, J., 2017. Is there a relationship between attitudes of shelter staff to cats and the cats' approach behaviour? Appl. Anim. Behav. Sci. 187, 60-68.

Bartlett, P.C., Bartlett, A., Walshaw, S., Halstead, S., 2005. Rates of euthanasia and adoption for dogs and cats in Michigan animal shelters. J. Appl. Anim. Welf. Sci . 8 (2), 97-104.

Biben, M., 1979. Predation and predatory play behaviour of domestic cats. Anim. Behav.27, 81-94.

Carlstead, K., Brown, J.L., Strawn, W., 1993a. Behavioral and physiological correlates of stress in laboratory cats. Appl. Anim. Behav. Sci. 38 (2), 143-158.

Carlstead, K., Brown, J.L., Seidensticker, J., 1993b. Behavioural and adrenocortical responses to environmental changes in leopard cats (Felis Bengalensis). Zoo Biol. 12 (4), 321-331.

Casey, R.A., Vandenbussche, S., Bradshaw, J.W., Roberts, M.A., 2009. Reasons for relinquishment and return of domestic cats (Felis silvestris catus) to rescue shelters in the UK. Anthrozos 22 (4), 347-358.

Demant, H., Ladewig, J., Balsby, T.J., Dabelsteen, T., 2011. The effect of frequency and duration of training sessions on acquisition and long-term memory in dogs. Appl. Anim. Behav. Sci. 133 (3), 228-234.

Dybdall, K., Strasser, R., 2014. Is there a bias against stray cats in shelters? People's perception of shelter cats and how it influences adoption time. Anthrozos 27 (4), 603-614.

Dybdall, K., Strasser, R., Katz, T., 2007. Behavioural differences between owner surrender and stray domestic cats after entering an animal shelter. Appl. Anim. Behav. Sci. 104 (1), 85-94. 
Fantuzzi, J.M., Miller, K.A., Weiss, E., 2010. Factors relevant to adoption of cats in an animal shelter. J. Appl. Anim. Welf. Sci. 13 (2), 174-179.

Finan, J.L., Taylor, L.F., 1940. Quantitative studies in motivation. I. Strength of conditioning in rats under varying degrees of hunger. J. Comp. Psychol. 29 (1), 119.

Fisher, G.T., 2009. The Thinking Dog: Crossover to Clicker Training. Dogwise Publishing.

Gourkow, N., 2001. Factors Affecting the Welfare and Adoption Rate of Cats in an Animal Shelter. Doctoral dissertation. University of British Columbia.

Gourkow, N., Fraser, D., 2006. The Effect of Housing and Handling Practices on the Welfare, Behaviour and Selection of Domestic Cats (Felis Sylvestris Catus) by Adopters in an Animal Shelter.

Gourkow, N., Hamon, S.C., Phillips, C.J., 2014a. Effect of gentle stroking and vocalization on behaviour, mucosal immunity and upper respiratory disease in anxious shelter cats. Prev. Vet. Med. 117 (1), 266-275.

Gourkow, N., LaVoy, A., Dean, G.A., Phillips, C.J.C., 2014b. Associations of behaviour with secretory immunoglobin A and cortisol in domestic cats during their first week in an animal shelter. Appl. Anim. Behav. Sci. 150, 55-64.

Gouveia, K., Magalhães, A., de Sousa, L., 2011. The behaviour of domestic cats in a shelter: Residence time, density and sex ratio. Appl. Anim. Behav. Sci. 130 (1), 53-59.

Herron, M.E., Shofer, F.S., Reisner, I.R., 2009. Survey of the use and outcome of confrontational and nonconfrontational training methods in client-owned dogs showing undesired behaviors. Appl. Anim. Behav. Sci. 117 (1), 47-54.

Hiby, E.F., Rooney, N.J., Bradshaw, J.W., 2006. Behavioural and physiological responses of dogs entering re-homing kennels. Physiol. Behav. 89 (3), 385-391.

Kelleher, R.T., Gollub, L.R., 1962. A review of positive conditioned reinforcement. J. Exp. Anal. Behav. 5 (S4), 543-597.

Kemp, I.R., Kaada, B.R., 1975. The relation of hippocampal theta activity to arousal, attentive behaviour and somato-motor movements in unrestrained cats. Brain Res. 95 (2-3), 323-342.

Kessler, M.R., Turner, D.C., 1997. Stress and adaptation of cats (Felis silvestris catus) housed singly, in pairs and in groups in boarding catteries. Anim. Welf. 6 (3), 243-254.

Kogan, L., Kolus, C., Schoenfeld-Tacher, R., 2017. Assessment of clicker training for shelter cats. Animals $7(10), 73$.

Laule, G., Desmond, T., 1998. Positive Reinforcement Training As an Enrichment Strategy. Smithsonian Institution, DC.

Lepper, M., Kass, P.H., Hart, L.A., 2002. Prediction of adoption versus euthanasia among dogs and cats in a California animal shelter. J. Appl. Anim. Welf. Sci . 5 (1), 29-42.

Levy, J.K., Crawford, P.C., 2004. Humane strategies for controlling feral cat populations. J. Am. Vet. Med. Assoc. 225 (9), 1354-1360.

Ley, J., 2015. Cats: not solitary but not really a social species. Feline Behavioral Health and Welfare-EBook. pp. 441.

Lindsay, S.R., 2013. Handbook of Applied Dog Behavior and Training, Adaptation and Learning. John Wiley \& Sons.

Luescher, A.U., Medlock, R.T., 2009. The effects of training and environmental alterations on adoption success of shelter dogs. Appl. Anim. Behav. Sci. 117 (1), 63-68. 
Mallapur, A., Qureshi, Q., Chellam, R., 2002. Enclosure design and space utilization by Indian leopards (Panthera pardus) in four zoos in southern India. J. Appl. Anim. Welf. Sci. 5 (2), 111-124.

McCune, S., Smith, C.P., Taylor, V., Nicol, C., 1995. Enriching the Environment of the Laboratory Cat. Environmental Enrichment Information Resources for Laboratory Animals: 1965-1995 Birds, Cats, Dogs, Farm Animals, Ferrets, Rabbits, and Rodents. pp. 27-42.

Melfi, V., 2013. Is training zoo animals enriching? Appl. Anim. Behav. Sci. 147 (3), 299-305.

Meyer, I., Ladewig, J., 2008. The relationship between number of training sessions per week and learning in dogs. Appl. Anim. Behav. Sci. 111 (3), 311-320.

Miller, P., 2001. Power of Positive Dog Training. Howell Book House.

Polín, E., Pérez, V., 2017. The effect of varied reinforcement on acquisition and extinction speed.

Psicothema 29 (1).

Pryor, K., 2009. Reaching the Animal Mind: Clicker Training and What It Teaches Us About All Animals. Simon and Schuster.

Rochlitz, I., 2000. Recommendations for the housing and care of domestic cats in laboratories. Lab. Anim. 34 (1), 1-9.

Ross, S.R., Schapiro, S.J., Hau, J., Lukas, K.E., 2009. Space use as an indicator of enclosure appropriateness: a novel measure of captive animal welfare. Appl. Anim.

Behav. Sci. 121 (1), 42-50.

Rushen, J., Taylor, A.A., de Passillé, A.M., 1999. Domestic animals' fear of humans and its effect on their welfare. Appl. Anim. Behav. Sci. 65 (3), 285-303.

Salman, M.D., New Jr., J.G., Scarlett, J.M., Kass, P.H., Ruch-Gallie, R., Hetts, S., 1998. Human and animal factors related to relinquishment of dogs and cats in 12 selected animal shelters in the United States. J. Appl. Anim. Welf. Sci. 1 (3), 207-226.

Salman, M.D., Hutchison, J., Ruch-Gallie, R., Kogan, L., New Jr., J.C., Kass, P.H., Scarlett, J.M., 2000. Behavioural reasons for relinquishment of dogs and cats to 12 shelters. J. Appl. Anim. Welf. Sci. 3 (2), 93106.

Savastano, G., Hanson, A., McCann, C., 2003. The development of an operant conditioning training program for New World primates at the Bronx Zoo. J. Appl. Anim. Welf. Sci. 6 (3), 247-261.

Scarlett, J.M., Donoghue, S., Saidla, J., Wills, J., 1994. Overweight cats: prevalence and risk factors. Int. J. Obesity Relat. Metab. Disorders 18, S22-8.

Scarlett, J.M., Salman, M.D., New Jr., J.G., Kass, P.H., 1999. Reasons for relinquishment of companion animals in US animal shelters: selected health and personal issues. J. Appl. Anim. Welf. Sci. 2 (1), 41-57.

Selman, L.D.A.M., 2017. The Effect of a Hiding Box on Stress Levels, Urinary Parameters, Body Weight, fURI and Adoption Rates in Dutch Shelter Cats (Master's thesis).

Shepherdson, D.J., Carlstead, K., Mellen, J.D., Seidensticker, J., 1993. The influence of food presentation on the behavior of small cats in confined environments. Zoo Biol. 12 (2), 203-216.

Shore, E.R., Petersen, C.L., Douglas, D.K., 2003. Moving as a reason for pet relinquishment: a closer look. J. Appl. Anim. Welf. Sci. 6 (1), 39-52. 
Slingerland, L.I., Fazilova, V.V., Plantinga, E.A., Kooistra, H.S., Beynen, A.C., 2009. Indoor confinement and physical inactivity rather than the proportion of dry food are risk factors in the development of feline type 2 diabetes mellitus. Vet. J. 179 (2),247-253.

Stanton, L.A., Sullivan, M.S., Fazio, J.M., 2015. A standardized ethogram for the felidae: A tool for behavioral researchers. Appl. Anim. Behav. Sci. 173, 3-16.

Tanaka, A., Wagner, D.C., Kass, P.H., Hurley, K.F., 2012. Associations among weight loss, stress, and upper respiratory tract infection in shelter cats. J. Am. Vet. Med. Assoc. 240 (5), 570-576.

Uetake, K., Goto, A., Koyama, R., Kikuchi, R., Tanaka, T., 2013. Effects of single caging and cage size on behavior and stress level of domestic neutered cats housed in an animal shelter. Anim. Sci. J. 84 (3), 272274.

Vinke, C.M., Godijn, L.M., Van der Leij, W.J.R., 2014. Will a hiding box provide stress reduction for shelter cats? Appl. Anim. Behav. Sci. 160, 86-93.

Ward, S.J., Melfi, V., 2013. The implications of husbandry training on zoo animal response rates. Appl. Anim. Behav. Sci. 147 (1), 179-185.

Wells, D.L., 2009. Sensory stimulation as environmental enrichment for captive animals

K. Westlund. 2014. Training is enrichment-and beyond. Appl. Anim. Behav. Sci., 152 (2014), pp. 1-6 